\title{
Virtual charge state separator as an advanced tool coupling measurements and simulations
}

\author{
S. Yaramyshev, ${ }^{*}$ H. Vormann, A. Adonin, W. Barth, L. Dahl, P. Gerhard, L. Groening, \\ R. Hollinger, M. Maier, S. Mickat, and A. Orzhekhovskaya \\ GSI Helmholtzzentrum für Schwerionenforschung, GmbH Planckstraße 1, 64291 Darmstadt, Germany
}

(Received 29 October 2014; published 29 May 2015)

\begin{abstract}
A new low energy beam transport for a multicharge uranium beam will be built at the GSI High Current Injector (HSI). All uranium charge states coming from the new ion source will be injected into GSI heavy ion high current HSI Radio Frequency Quadrupole (RFQ), but only the design ions $\mathrm{U}^{4+}$ will be accelerated to the final RFQ energy. A detailed knowledge about injected beam current and emittance for pure design $\mathrm{U}^{4+}$ ions is necessary for a proper beam line design commissioning and operation, while measurements are possible only for a full beam including all charge states. Detailed measurements of the beam current and emittance are performed behind the first quadrupole triplet of the beam line. A dedicated algorithm, based on a combination of measurements and the results of advanced beam dynamics simulations, provides for an extraction of beam current and emittance values for only the $\mathrm{U}^{4+}$ component of the beam. The proposed methods and obtained results are presented.
\end{abstract}

DOI: 10.1103/PhysRevSTAB.18.050103

PACS numbers: 29.20.Ej, 41.75.Ak, 41.75.Cn, 45.50.Dd

\section{INTRODUCTION}

A low energy beam transport line (LEBT) for the multicharged ion beams usually comprises a dipole bending magnet(s). This allows for the separation and further acceleration of the design charge state only. However, a straight beam transport line without a dipole(s) and the injection of multicharged ion beams into an RFQ accelerator are under consideration worldwide [1-3]. A new straight LEBT for the multicharged uranium beam will be integrated into the operating GSI heavy ion high current linac UNILAC [4].

\section{HIGH CURRENT HEAVY ION UNILAC}

Besides two ion source terminals and a LEBT, the High Current Injector (HSI) of the GSI UNILAC (Fig. 1) is comprised of a $36 \mathrm{MHz}$ RFQ accelerating the ion beam from $2.2 \mathrm{keV} / \mathrm{u}$ up to $120 \mathrm{keV} / \mathrm{u}$ and a short RFQ adapter. The $36 \mathrm{MHz}$ Interdigital H-mode Drift Tube Linac (IH DTL), consisting of two separate tanks, accelerates the beam up to the energy of $1.4 \mathrm{MeV} / \mathrm{u}$. After stripping and charge state separation, the $108 \mathrm{MHz}$ Alvarez DTL provides for beam acceleration up to $11.4 \mathrm{MeV} / \mathrm{u}$. The transfer line (TK) to the synchrotron SIS 18 is equipped with a stripper and charge state separator system [5].

A new ion source terminal and new straight LEBT, which are mainly foreseen for operation with uranium beams, are under development now. All uranium charge

\footnotetext{
*s.yaramyshev@gsi.de

Published by the American Physical Society under the terms of the Creative Commons Attribution 3.0 License. Further distribution of this work must maintain attribution to the author $(s)$ and the published article's title, journal citation, and DOI.
}

states coming from the new ion source will be injected into HSI RFQ, but only design ions $\mathrm{U}^{4+}$ will be accelerated to the final RFQ energy of $120 \mathrm{keV} / \mathrm{u}$ [6].

\section{THE MEASUREMENTS CAMPAIGN}

An intense experimental campaign was carried out in June-November 2013 at the existing north terminal (Fig. 2) of UNILAC. A set of beam current and emittance measurements behind the first quadrupole triplet of the existing LEBT have been performed. The beam current and transverse emittance obtained with different settings of the ion source terminal were at that time in the range of $20-35 \mathrm{~mA}$ inside $300-450 \mathrm{~mm} * \mathrm{mrad}$, respectively [7]. These measurements have been performed with the goal to upgrade and optimize the heavy ion high current UNILAC as an injector for Facility for Antiproton and Ion Research at Darmstadt (FAIR) [8].

Usually, an intense uranium beam, coming from the existing ion source terminal, mainly consists of $3+$ and $4+$ ions, while contributions from other charge states are relatively small. Figure 3 represents a typical intensity distribution for different uranium charge states, measured at the existing LEBT downstream of the $77.5^{\circ}$ dipole.

\section{BEAM DYNAMICS SIMULATIONS}

As shown in Fig. 4(a), a different focusing strength for the different charge states leads to a complicated shape of the composite beam emittance, measured behind the quadrupole triplet. Obviously, standard diagnostics are not able to distinguish ions with different charge states. Nevertheless, a macroparticle distribution in 2D vertical phase space [Fig. 4(b)] was generated from the raw data of the 


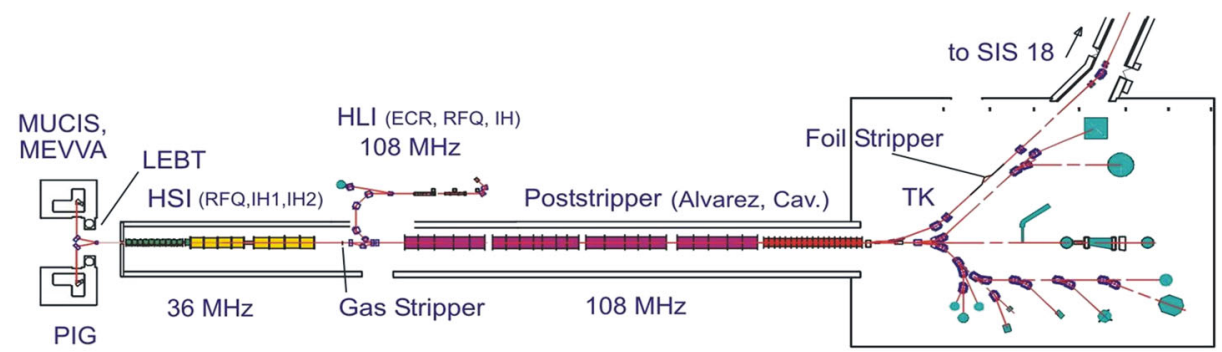

FIG. 1. Schematic overview of the GSI UNILAC.

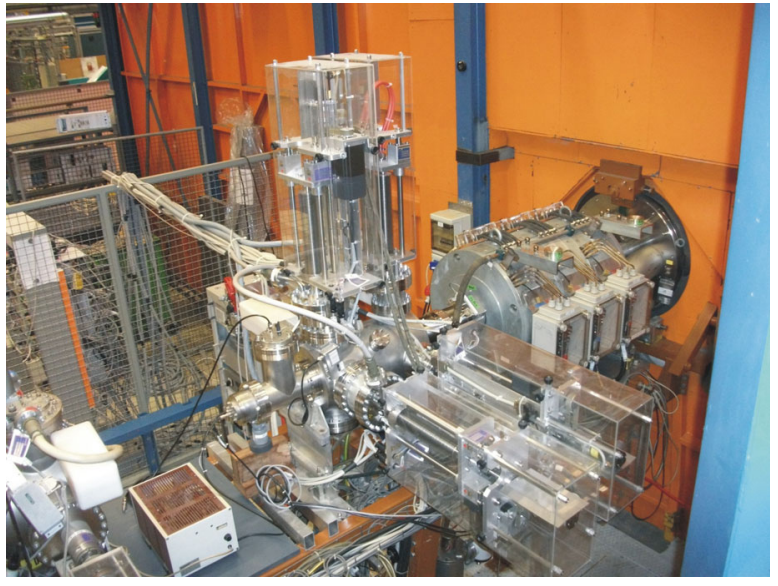

FIG. 2. The experimental setup for the measurements of beam current and emittance.

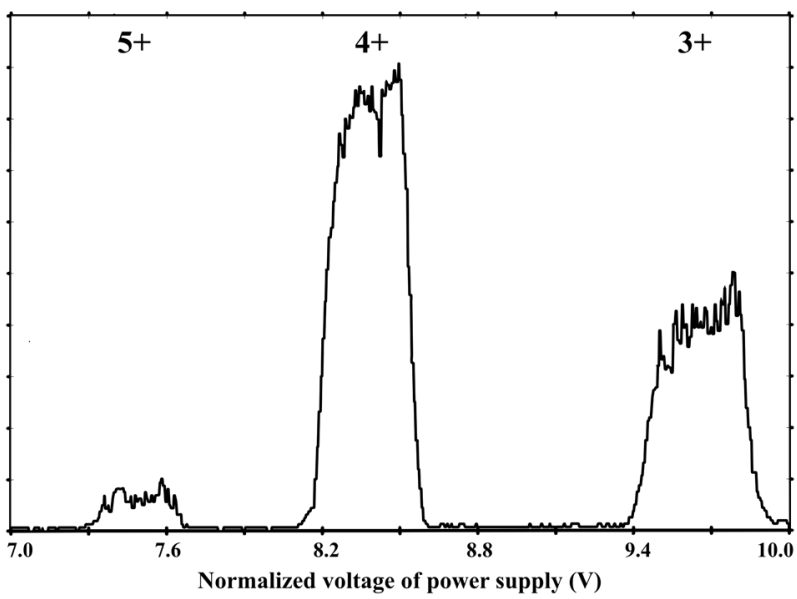

FIG. 3. Measured intensity distribution for different uranium charge states, coming from the existing ion source terminal.

measured emittance. The density of macroparticles is proportional to the local intensity, measured with a slit-grid device inside each bin $0.5 \mathrm{~mm} \times 1.7 \mathrm{mrad}$. The same procedure has been implemented for the horizontal measurements.

A 4D transverse particle distribution has been created, assuming an elliptical beam shape in real space of coordinates and velocities. A continuous beam has been assumed for the longitudinal phase space [9].
Based on the experimental experience and previous simulation results [10-13], a full neutralization of the space charge along the LEBT was assumed.

A set of beam dynamics simulations have been performed by means of the macroparticle code DYNAMION [14]. As the measured uranium beam emittance is mainly formed by charge states $4+$ and $3+$, the beam dynamics was separately simulated for two identical $6 \mathrm{D}$ phase space particle distribution, but with a different charge state $(4+$ or $3+)$ assigned to all particles. These two input distributions have been transported backward (upstream) through the quadrupole triplet to the same position at the ion source exit [Fig. 5(a)].

The magnetic field along the triplet has been represented by 3D mapping and constructed inside the code DYNAMION from detailed magnetic field measurements for each quadrupole separately. An overlapping of the magnetic field from neighboring lenses is included automatically. The gradients of the lenses (Table I) have been calculated from the machine settings and from the measured data for the magnetic field of each quadrupole.

The two particle distributions are transported through the magnetic quadrupoles with trajectories dependent on the charge state and form different beam emittances at the same position at the ion source exit [Fig. 5(a)]. But in assumption that the beam parameters behind a Vacuum ARc Ion Source (VARIS) ion source are the same for every charge state, only a phase space that overlaps the different particle distributions could be treated as an original emittance formed by the complete beam. Only the bins which include simultaneously $\mathrm{U}^{3+}$ and $\mathrm{U}^{4+}$ macroparticles were considered. The selected macroparticles are shown on Fig. 5(b).

The obtained "realistic" particle ensemble has been simulated forward (downstream) through the quadrupole triplet with the same settings, again separately as $\mathrm{U}^{3+}$ and as $\mathrm{U}^{4+}$. The transported particles represent at the position of measurements two separate beam emittances for each charge state $\mathrm{U}^{3+}$ and $\mathrm{U}^{4+}$ (Fig. 6). These two macroparticle ensembles together cover well the intricate shape of the original distribution generated from the measured emittance.

As expected, a significant part of the beam emittance behind quadrupole triplet is formed by the nonuniform mixture of $\mathrm{U}^{3+}$ and $\mathrm{U}^{4+}$ ions, while some areas are covered by only one charge state. 

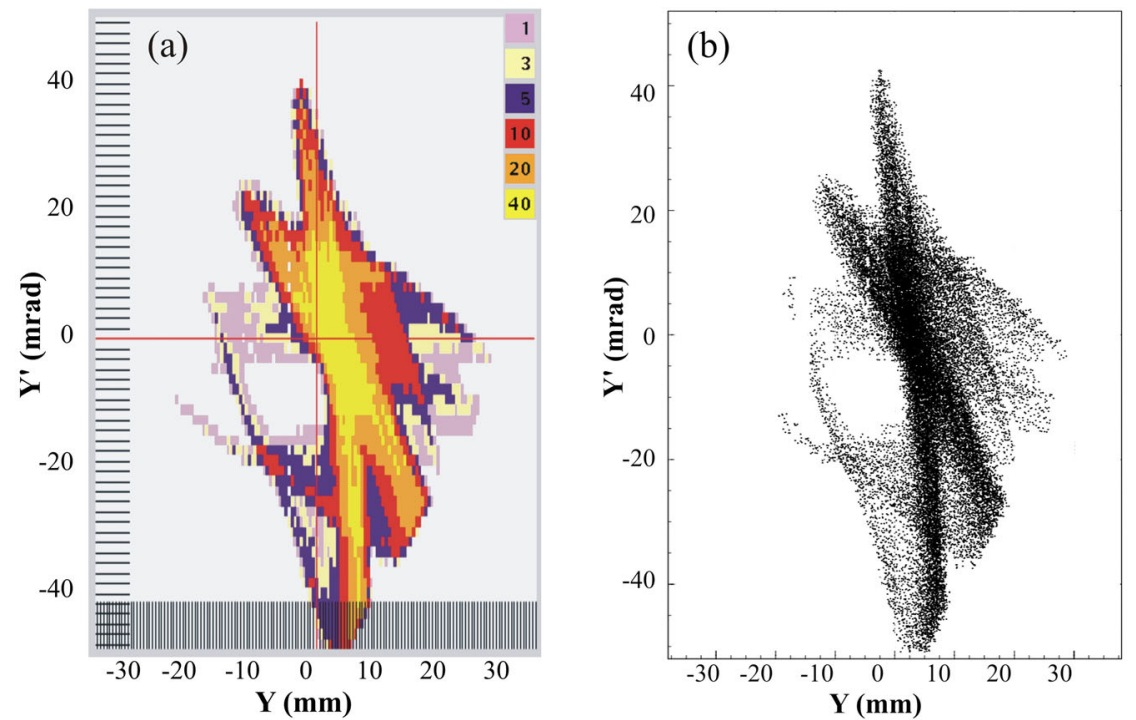

FIG. 4. (a) The screen shot of measured beam emittance in the vertical phase plane. Different colors represent the range of intensities. (b) The macroparticle distribution in the vertical phase plane generated from the measured emittance which is shown in (a).
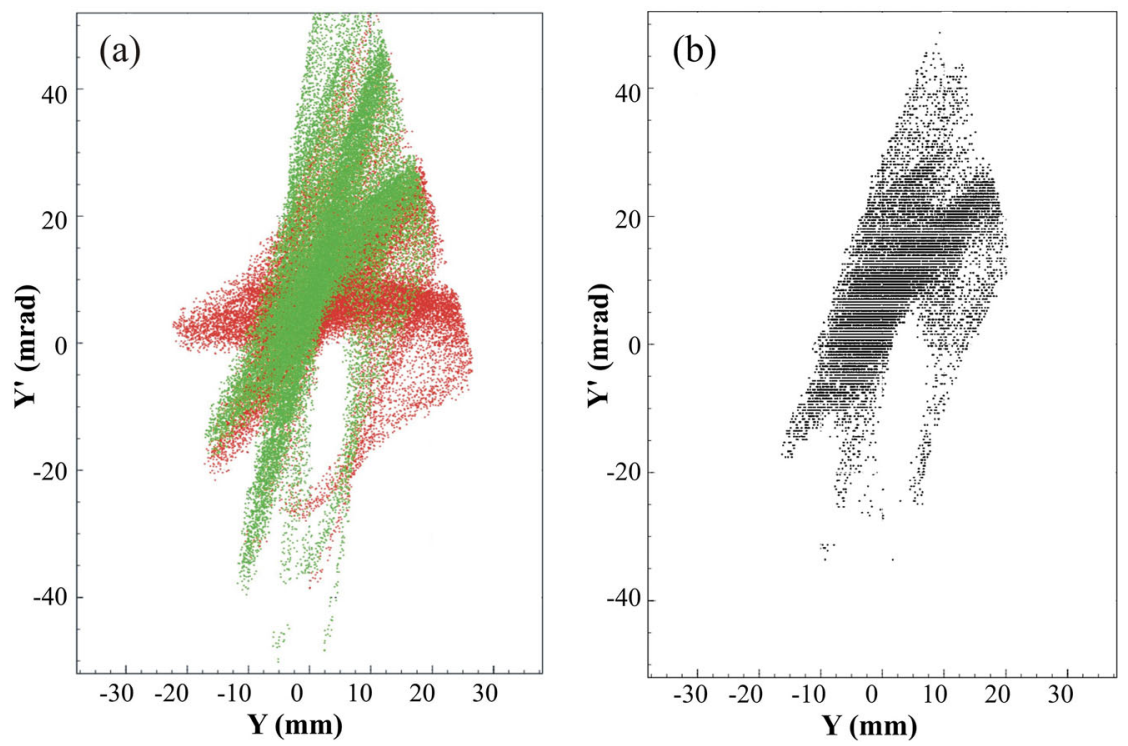

FIG. 5. (a) The macroparticle distributions in the vertical phase plane transported backward through the quadrupole triplet as $\mathrm{U}^{4+}$ (green) and as $\mathrm{U}^{3+}$ (red) to the same position at the ion source exit. (b) The overlapping of the macroparticle distributions which are shown in (a).

\section{AN INTENSITY RATIO}

Generally, a uranium beam from an ion source is comprised of different charge states: $\mathrm{U}^{1+}+\mathrm{U}^{2+}+\mathrm{U}^{3+}+$ $\mathrm{U}^{4+}+\mathrm{U}^{5+}+\mathrm{U}^{6+}$. The amount of each charge state

TABLE I. Magnetic field gradients for the triplet.

\begin{tabular}{lcc}
\hline \hline Quadrupole & Wire coil current $(\mathrm{A})$ & Gradient $(\mathrm{T} / \mathrm{m})$ \\
\hline 1 & 70.8 & +9.29 \\
2 & 137.6 & -14.20 \\
3 & 46.3 & +6.14 \\
\hline \hline
\end{tabular}

depends on multiparametric settings of the ion source and postacceleration gap.

The beam currents and beam emittances are measured downstream of the magnetic quadrupole triplet for a mixture of uranium charge states (mostly $3+$ and $4+$ ). Obviously, a current transformer, as well as an emittance scanner, is unable to distinguish different charge states. But, especially for the new UNILAC-LEBT with straight beam injection into the RFQ (without charge states separation by dipole), information about the beam current and emittance for the design ion $\mathrm{U}^{4+}$ is essential. 


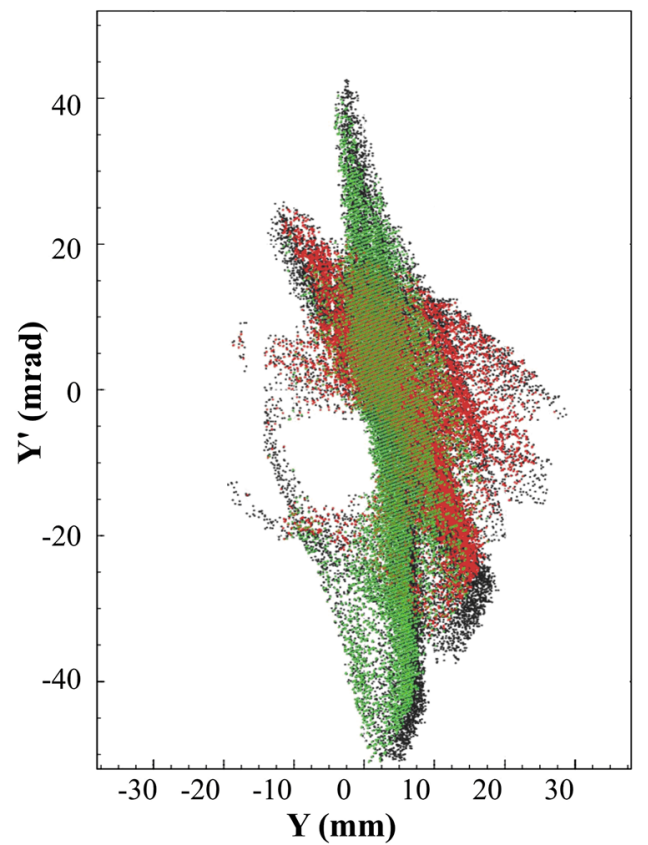

FIG. 6. The macroparticle distributions in the vertical phase plane generated from the emittance measurement (black) and simulated ones for $\mathrm{U}^{4+}$ (green) and $\mathrm{U}^{3+}$ (red) ions.

The beam emittances have been measured with a slit-grid device. As raw data, one obtains a local beam intensity for each bin (phase square) in some range of coordinates and angle. The measured local intensity in each bin is the sum of all charge states $\boldsymbol{k}+$ :

$$
I_{j}=\sum_{k+} I_{j}{ }^{k+} .
$$

Because of a different focusing of uranium charge states by the quadrupole triplet, the contribution of each charge state to a local measured intensity $\boldsymbol{I}_{\boldsymbol{j}}$ varies significantly from bin to bin. This effect provides the possibility to estimate a ratio between different charge states, although the measurements are performed for the complete beam.

The number of macroparticles for a beam dynamics simulation (for each uranium charge state separately or all charge states together) is a fixed number. However, an unknown ratio of the real intensities of different charge states cannot be represented in simulations by a different number of particles in advance. Nevertheless, as a result of simulations, one can count inside the bin $\boldsymbol{j}$ the number of macroparticles $N_{j}^{k+}$ for each charge state $\boldsymbol{k}+$. The total number of simulated macroparticles with different charge states inside the bin $\boldsymbol{j}$ is

$$
N_{j}=\sum_{k+} N_{j}^{k+}
$$

and varies significantly from bin to bin, in the same way as real contributions of each charge state to the measured local intensity at each bin. Therefore, the measured intensity in each bin can be expressed as

$$
I_{j}=\sum_{k+} x_{k+} N_{j}{ }^{k+},
$$

where $\boldsymbol{x}_{\boldsymbol{k}+}$ are the unknown coefficients for contributions of charge state $\boldsymbol{k}+$ to the total beam intensity.

\section{A. An intensity ratio by least squares}

Taking into account only two charge states $\mathrm{U}^{3+}$ and $\mathrm{U}^{4+}$, a direct enumeration for an intensity ratio has been performed.

The total beam intensity has been measured in front of an emittance scanner. The data, measured with a slit-grid device, are represented by a local beam intensity at each phase space bin $(0.5 \mathrm{~mm} \times 1.7 \mathrm{mrad})$. Following the given assumption, this measured local intensity inside a bin is comprised by two charge states $\mathrm{U}^{4+}$ and $\mathrm{U}^{3+}$.

The results of beam dynamics simulations are represented by macroparticle distribution $\left(\mathrm{U}^{4+}\right.$ and $\left.\mathrm{U}^{3+}\right)$ at the position of emittance measurements. Therefore, a number of macroparticles of each charge state at every bin is known.

Assuming $\mathrm{U}^{4+}$ beam intensity of a given level of the measured total one, a corresponding portion of intensity could be assigned to each simulated $\mathrm{U}^{4+}$ macroparticle. The rest of the measured total intensity is assigned to simulated $\mathrm{U}^{3+}$ macroparticles in the same way. Then the least squares for the assigned intensity $\boldsymbol{C}_{\boldsymbol{j}}$ of the macroparticles inside a bin $\boldsymbol{j}$ and the measured local intensity $\boldsymbol{I}_{\boldsymbol{j}}$ at the same bin are calculated for all bins:

$$
\Delta=\frac{1}{J} \sqrt{\sum_{j=1}^{J}\left(C_{j}-I_{j}\right)^{2}} .
$$

Such a scan for assumed $\mathrm{U}^{4+}$ intensity from $1 \%$ to $100 \%$ of the total one leads to a smooth optimum at a level of $60 \%-65 \%$ (Fig. 7).

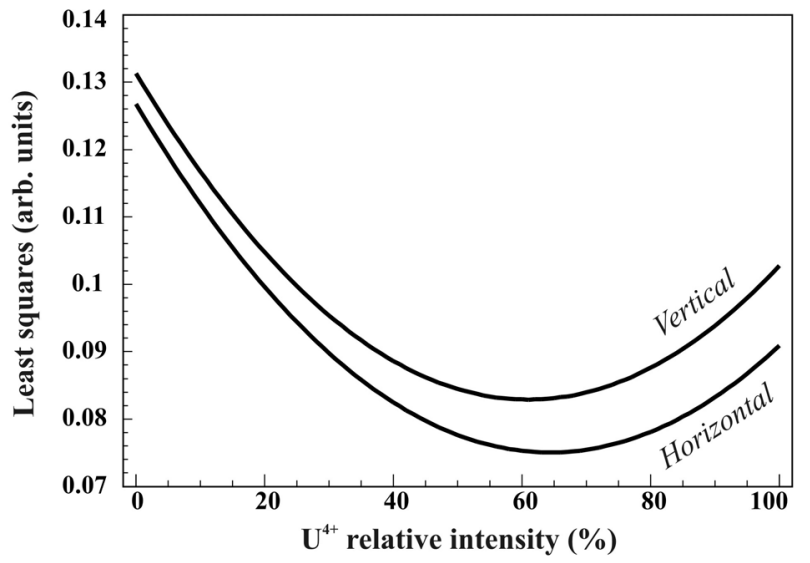

FIG. 7. The least squares direct enumeration assuming given a total ratio of $\mathrm{U}^{4+}$ intensity from $1 \%$ to $100 \%$. 


\section{B. An intensity ratio by matrix equation}

Let us consider an equation:

$$
\begin{aligned}
A(J, K) X(K) & =B(J), \quad \text { where } \\
A(J, K) & =\left(\begin{array}{lll}
a_{11} & \ldots & a_{1 K} \\
\ldots & \ldots & \ldots \\
a_{J 1} & \ldots & a_{J K}
\end{array}\right), \\
X(K) & =\left(\begin{array}{c}
x_{1} \\
\\
x_{K}
\end{array}\right), \quad \text { and } \\
B(J) & =\left(\begin{array}{c}
b_{1} \\
\ldots \\
b_{J}
\end{array}\right)
\end{aligned}
$$

are a matrix of coefficients, a vector of variables, and a vector of meanings, correspondingly.

Then a well-known solution [15] of such an overdefined linear system is

$$
P=\left(A^{T} A\right)^{-1} A^{T} B
$$

For this case, $\boldsymbol{a}_{\boldsymbol{j} \boldsymbol{k}}$ is a number of simulated particles with charge state $\boldsymbol{k}$ inside bin $\boldsymbol{j}, \boldsymbol{b}_{\boldsymbol{j}}$ the measured intensity in bin $\boldsymbol{j}$, and $\boldsymbol{x}_{\boldsymbol{k}}$ the relative total intensity of charge state $\boldsymbol{k}$.

On the basis of an experimental experience (Fig. 3) and the simulation results (Fig. 6), only two charge states $3+$ and $4+$ have been considered. The intensity of all other charge states is assumed as negligible. In this case, the matrix transforms to $\boldsymbol{A}(\boldsymbol{J}, \boldsymbol{2})$, where $\boldsymbol{J}$ is the number of bins with a nonzero quantity of simulated backward-forward $\mathrm{U}^{3+}$ or $\mathrm{U}^{4+}$ macroparticles.

Two transverse phase planes $X-X^{\prime}$ and $Y-Y^{\prime}$ were under consideration independently. Two "solutions" of such overdefined linear systems give the most probable intensity ratio of $\mathrm{U}^{3+}$ and $\mathrm{U}^{4+}$ for the measured beam emittances (Table II).

Also, an evaluation of the horizontal and vertical measurements together has been performed, and a coincidence in the range of a few percent has been reached. As expected, the obtained results by the matrix solution are in a good agreement with direct enumeration by the least squares method.

TABLE II. Calculated relative intensity.

\begin{tabular}{lll}
\hline \hline Phase plane & $\mathrm{U}^{4+}$ & $\mathrm{U}^{3+}$ \\
\hline Vertical & $64 \%$ & $36 \%$ \\
Horizontal & $61 \%$ & $39 \%$ \\
\hline \hline
\end{tabular}

\section{SUMMARY AND CONCLUSION}

A dedicated method to distinguish between different charge states coming from an ion source has been proposed, developed, and realized. The macroparticle distributions have been generated from the raw data of an emittance measurements, taking into account details of the intensity distribution inside the 4D transverse phase space. Accurately measured distributions of the magnetic field along the quadrupole triplet are used to produce the realistic results of beam dynamics simulations.

Using the proposed method, one can extract from the measurements for the mixed uranium beam, which comprises different charge states (for a recent case, mainly $\mathrm{U}^{3+}$ and $\mathrm{U}^{4+}$ ), the detailed macroparticle distribution (which represents beam emittance) and beam intensity for the design ion $\mathrm{U}^{4+}$ only. Additionally, a matrix algorithm, based on the solution of an overdefined system of linear equations, could provide for an estimated $\mathrm{U}^{4+}$ intensity, while measurements could be done for all uranium charge states together $(1+, 2+, 3+, 4+, 5+$, and $6+)$.

Generally, the proposed coupling of detailed measurements and precise beam dynamics simulations with the code DYNAMION acts as a virtual charge state separator. Finally, it provides beam parameters which cannot be measured directly with standard beam diagnostics.

Further development of the proposed algorithm under space charge conditions is recently under investigation.

The information obtained by this method has been used for the final layout of the new GSI heavy ion LEBT for a straight injection of the beam into the HSI RFQ. The developed procedure could be implemented for further investigation and optimization of the existing UNILAC beam lines.

The proposed algorithm might have a particular interest worldwide [16-19] for the design, optimization, and operation of a transport line for multicharged beams.

\section{ACKNOWLEDGMENTS}

The authors thank GSI colleagues from various departments (ion sources; linacs; mechanics, transport, and installations; electric power converters; beam instrumentation; accelerator controls), who cooperatively supported and performed the field measurements for the quadrupoles, the assembly work, the cabling and calibrating of the beam diagnostics, the connection of the temporary device to the UNILAC controls, and the operation of the experiment.

[1] V. Andreev, A. Kolomiets, S. Minaev, V. Pershin, T. Tretjakova, R. Vengrov, I. Vorobjov, and S. Yaramyshev, in Proceedings of the 6th European Particle Accelerator Conference, Stockholm, 1998 (IOP, London, 1998), p. $755 \mathrm{x}$. 
[2] P. N. Ostroumov, R. C. Pardo, G. P. Zinkann, K. W. Shepard, and J. A. Nolen, Phys. Rev. Lett. 86, 2798 (2001).

[3] M. Okamura, T. Katayama, R. A. Jameson, T. Takeuchi, and T. Hattori, Rev. Sci. Instrum. 73, 761 (2002).

[4] L. Dahl, W. Barth, and S. Yaramyshev, in Proceedings of the 21st International Linac Conference, Gyeongju, Korea, 2002 (Pohang Accelerator Laboratory, Pohang, Korea, 2002), p. 352.

[5] H. Vormann, S. Yaramyshev, A. Adonin, W. Barth, L. Dahl, P. Gerhard, L. Groening, R. Hollinger, M. Maier, S. Mickat, A. Orzhekhovskaya, and C. Xiao, in Proceedings of 5 th International Particle Accelerator Conference, Dresden, Germany, 2014, http://jacow.org/.

[6] W. Barth, W. Bayer, L. Dahl, L. Groening, S. Richter, and S. Yaramyshev, Nucl. Instrum. Methods Phys. Res., Sect. A 577, 211 (2007).

[7] S. Yaramyshev, H. Vormann, A. Adonin, W. Barth, R. Berezov, L. Dahl, P. Gerhard, L. Groening, R. Hollinger, M. Maier, S. Mickat, A. Orzhekhovskaya, and C. Xiao, GSI Annual Report 2013, Darmstadt, Germany, p. 291.

[8] W. Barth, B. Schlitt, G. Clemente, W. Vinzenz, S. Mickat, and L. Dah, in Proceedings of the 2nd International Particle Accelerator Conference, San Sebastián, Spain (EPS-AG, Spain, 2011), p. 2550.

[9] S. Yaramyshev, H. Vormann, A. Adonin, W. Barth, L. Dahl, P. Gerhard, L. Groening, R. Hollinger, M. Maier, S. Mickat, and A. Orzhekhovskaya, in Proceedings of 5th International Particle Accelerator Conference, Dresden, Germany, 2014, http://jacow.org/.

[10] N. Chauvin, O. Delferrière, R. Duperrier, and R. Gobin, in Proceedings of the 25th International Linear Accelerator
Conference, LINAC-2010, Tsukuba, Japan (KEK, Tsukuba, Japan, 2010), p. 740.

[11] P. Spaedtke, Rev. Sci. Instrum. 85, 02 A744 (2014).

[12] R. Scrivens, G. Bellodi, O. Crettiez, V. Dimov, D. Gerard, E. Granemann Souza, R. Guida, J. Hansen, J.-B. Lallement, J. Lettry, A. Lombardi, Ø. Midttun, C. Pasquino, U. Raich, B. Riffaud, F. Roncarolo, C. A. Valerio-Lizarraga, J. Wallner, M. Yarmohammadi Satri, and T. Zickler, Rev. Sci. Instrum. 85, 02A729 (2014).

[13] Y. K. Batygin, C. Pillai, and L. J. Rybarcyk, in Proceedings of 5th International Particle Accelerator Conference, Dresden, Germany, 2014, http://jacow.org/.

[14] S. Yaramyshev, W. Barth, L. Groening, A. Kolomiets, and T. Tretyakova, Nucl. Instrum. Methods Phys. Res., Sect. A 558, 90-94 (2006).

[15] G. Strang, Linear Algebra and Its Applications (Mir, Moscow, 1980).

[16] B. Yu. Sharkov, N. N. Alexeev, M. D. Churazov, A. A. Golubev, D. G. Koshkarev, and P. R. Zenkevich, Nucl. Instrum. Methods Phys. Res., Sect. A 464, 1-5 (2001).

[17] J. Tamura et al., in Proceedings of 1st Heavy Ion Accelerator Technology Conference, Venice, Italy, 2009, http://jacow.org/.

[18] L. Weissman, D. Berkovits, S. Halfon, and Y. Yanay, in Proceedings of the 25th International Linear Accelerator Conference, LINAC-2010, Tsukuba, Japan (KEK, Tsukuba, Japan, 2010), p. 584.

[19] A. Kovalenko and A. Kolomiets, in Proceedings of 20st Linear Accelerator Conference, Tel-Aviv, Israel, 2012, http://jacow.org/. 